\title{
Análise da Revisão Cochrane: Alta Hospitalar Precoce para Hospitalização Domiciliária. Cochrane Database Syst Rev. 2017;6:CD000356.
}

\author{
Analysis of the Cochrane Review: Early Discharge Hospital \\ at Home. Cochrane Database Syst Rev. 2017;6:CD000356.
}

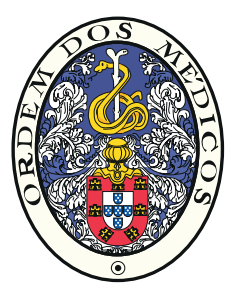

Mariana ALVES* $\triangle^{1,2}$, Miguel BIGOTTE VIEIRA*3, João COSTA ${ }^{4,5}$, António VAZ-CARNEIRO ${ }^{4,5}$

Acta Med Port 2017 Dec;30(12):835-839 - https://doi.org/10.20344/amp.9791

\begin{abstract}
RESUMO
A hospitalização domiciliária é um serviço que proporciona tratamento a doentes com doença aguda no próprio domicílio, os quais noutras condições necessitariam de internamento hospitalar em enfermaria de agudos. No entanto, o benefício clínico desta intervenção e o seu efeito nos custos em saúde não se encontram estabelecidos. Esta revisão sistemática Cochrane teve como objectivo avaliar a eficácia e custos associados aos cuidados de doentes admitidos no serviço de hospitalização domiciliária em comparação com internamento hospitalar habitual. Foi realizada revisão sistemática da literatura através de pesquisa nas seguintes bases de dados - The Cochrane Effective Practice and Organisation of Care Group (EPOC) register, Cochrane Central Register of Controlled Trials (CENTRAL), MEDLINE, Embase, CINAHL, e EconLit - até 9 de janeiro de 2017. Foram incluídos 32 ensaios clínicos aleatorizados (2 dos quais não publicados), incluindo 4746 doentes. A presente revisão detetou evidência insuficiente do benefício económico (através de uma redução no período de internamento hospitalar) ou melhoria dos resultados clínicos da hospitalização domiciliária.

Palavras-chave: Alta Hospitalar; Custos de Cuidados de Saúde; Mortalidade; Reinternamento; Revisão Sistemática; Serviços Hospitalares de Assistência Domiciliar
\end{abstract}

\section{ABSTRACT}

Hospital at home is a service that provides active treatment by healthcare professionals in the patient's home for a condition that otherwise would require acute hospital in-patient care. However, the clinical benefit of this intervention and its effect on health costs are not established. This Cochrane systematic review aimed to assess the effectiveness and costs of managing patients with hospital at home compared with inpatient hospital care. A systematic review of the literature was carried out by searching the following databases to 9 January 2017: Cochrane Effective Practice and Organization of Care Group (EPOC) register, Cochrane Central Register of Controlled Trials (CENTRAL), MEDLINE, Embase, CINAHL, EconLit and clinical trials registries. Thirty-two randomized trials (2 of which unpublished), including 4746 patients, were included. The present review provides insufficient objective evidence of economic benefit (through a reduction in hospital length of stay) or improved health outcomes.

Keywords: Health Care Costs; Home Care Services, Hospital-Based; Mortality; Patient Discharge; Patient Readmission; Systematic Review

\section{QUESTÃO CLÍNICA}

Qual é a efetividade e o impacto nos custos da alta precoce para o domicílio com apoio de serviço de hospitalização domiciliária?

\section{OBJETIVOS}

Primários:

- mortalidade;

- reinternamento hospitalar.

Secundários:

- status funcional;

- dados reportados pelo doente (incluindo bem estar psicológico, estado de saúde específico da doença, qualidade de vida e funcionamento cognitivo);

- complicações clínicas;

- institucionalização após a alta;

- satisfação do doente;
- opinião do cuidador (incluindo satisfação e sobrecarga);

- informação de profissionais de saúde (incluindo satisfação do médico de família);

- duração de internamento (incluindo número de dias de internamento e número de dias de cuidados médicos);

- utilização de recursos de saúde (incluindo custos de intervenções e tratamento habitual).

Foi realizada uma pesquisa da literatura, até 9 de Janeiro 2017, nas seguintes bases de dados: The Cochrane Effective Practice and Organisation of Care Group (EPOC) register, Cochrane Central Register of Controlled Trials (CENTRAL), MEDLINE, Embase, CINAHL e EconLit. Foram verificadas as referências bibliográficas dos artigos relevantes identificados na pesquisa. Foram pesquisadas as citações dos artigos incluídos da anterior revisão Cochrane do mesmo tema, utilizando a ferramenta

\footnotetext{
* Co-primeiros autores.

1. Serviço de Medicina III. Centro Hospitalar Lisboa Norte. Lisboa. Portugal.

2. Instituto de Semiótica Clínica. Faculdade de Medicina. Universidade de Lisboa. Lisboa. Portugal.

3. Serviço de Nefrologia e Transplantação Renal. Centro Hospitalar Lisboa Norte. Lisboa. Portugal.

4. Centro de Estudos de Medicina Baseada na Evidência. Faculdade de Medicina. Universidade de Lisboa. Lisboa. Portugal.

5. Cochrane Portugal. Lisboa. Portugal.

$\square$ Autor correspondente: Mariana Alves. marianaalves88@gmail.com

Recebido: 14 de outubro de 2017 - Aceite: 13 de dezembro de 2017| Copyright @ Ordem dos Médicos 2017
} 
Science Citation Index. Foi realizada ainda uma pesquisa nos websites de registos de ensaios clínicos utilizando o termo "hospital at home" (who.int/ictrp) e os termos "hospital at home" e "admissão" (ClinicalTrials.gov).

\section{METODOLOGIA}

Revisão sistemática da literatura de ensaios clínicos aleatorizados comparando alta precoce para hospitalização domiciliária versus internamento em enfermaria de doença aguda. Foram incluídos estudos que avaliassem doentes adultos ( $\geq 18$ anos) elegíveis para hospitalização domiciliária. Foram excluídos estudos que reportassem dados relativos a hospitais obstétricos, pediátricos e psiquiátricos, cuidados prestados em ambulatório, cuidados paliativos domiciliários, cuidados prestados no domicílio após a alta hospitalar, bem como internamentos em unidades de longa duração (ausência de doença aguda).

Relativamente ao tipo de intervenção, consideraram-se os cuidados de saúde prestados no domicílio do doente por diferentes instituições (serviços hospitalares, comunitários ou mistos).

\section{RESULTADOS}

Foram incluídos 32 ensaios clínicos aleatorizados (dois dos quais não publicados), incluindo 4746 doentes de 12 países. Onze estudos incluíram doentes em recuperação após acidente vascular cerebral (AVC), oito estudos incluíram doentes com várias comorbilidades, cinco estudos incluíram doentes com doença pulmonar obstrutiva crónica (DPOC), um estudo incluiu doentes com pancreatite aguda não alcoólica e um estudo incluiu doentes com insuficiência cardíaca. Estudos no contexto de recuperação pós cirurgia eletiva incluíram doentes com hérnias e varizes, bypass de artéria coronária, colocação de prótese de joelho e fratura da anca. Foram excluídos 34 estudos, 12 dos quais novos em relação à anterior revisão Cochrane do mesmo tema.

A maioria dos cuidados eram providenciados por equipa de enfermagem com apoio de outros profissionais (médicos,

Tabela 1 - Sumário de resultados nos doentes com AVC, adaptado da versão original

\begin{tabular}{|c|c|c|c|c|c|c|}
\hline \multicolumn{7}{|c|}{ Efeito da alta precoce para hospitalização domiciliária em doentes em recuperação após AVC } \\
\hline \multicolumn{7}{|c|}{$\begin{array}{l}\text { Doente ou população: doentes em recuperação após AVC, que precisariam de cuidados em internamento hospitalar } \\
\text { Contexto: Austrália, Canadá, Noruega, Suécia, Tailândia, Reino Unido } \\
\text { Intervenção: hospitalização domiciliária } \\
\text { Comparação: tratamento habitual }\end{array}$} \\
\hline \multirow[t]{2}{*}{ Resultados } & \multicolumn{2}{|c|}{$\begin{array}{l}\text { Riscos comparativos ilustrativos* } \\
\text { (IC 95\%) }\end{array}$} & \multirow[t]{2}{*}{$\begin{array}{l}\text { Efeito relativo } \\
\text { (IC 95\%) }\end{array}$} & \multirow{2}{*}{$\begin{array}{l}\text { Número de } \\
\text { participantes } \\
\text { (ensaios) }\end{array}$} & \multirow{2}{*}{$\begin{array}{l}\text { Qualidade da } \\
\text { evidência } \\
\text { (GRADE) }\end{array}$} & \multirow[t]{2}{*}{ Comentários } \\
\hline & $\begin{array}{l}\text { Ausência de alta } \\
\text { precoce para } \\
\text { hospitalização } \\
\text { domiciliária } \\
\text { (risco assumido) }\end{array}$ & $\begin{array}{l}\text { Alta precoce para } \\
\text { hospitalização } \\
\text { domiciliária (risco } \\
\text { correspondente) }\end{array}$ & & & & \\
\hline $\begin{array}{l}\text { Mortalidade } \\
\text { ( } 3 \text { a } 6 \text { meses })\end{array}$ & 56 por 1000 & $\begin{array}{l}52 \text { por } 1000 \\
(32 \text { a } 83)\end{array}$ & $\begin{array}{c}\text { RR } 0,92 \\
(0,57 \text { a } 1,48)\end{array}$ & $\begin{array}{l}1114 \\
(11)\end{array}$ & Moderada $^{1}$ & \\
\hline $\begin{array}{l}\text { Reinternamento hospitalar } \\
\text { ( } 3 \text { a } 6 \text { meses) }\end{array}$ & 187 por 1000 & $\begin{array}{l}204 \text { por } 1000 \\
(133 \text { a } 211)\end{array}$ & $\begin{array}{c}\text { RR } 1,09 \\
(0,71 \text { a } 1,66)\end{array}$ & $\begin{array}{c}345 \\
(5)\end{array}$ & Baixa $^{2}$ & \\
\hline $\begin{array}{l}\text { Institucionalização } \\
\text { ( } 3 \text { a } 6 \text { meses) }\end{array}$ & 150 por 1000 & $\begin{array}{l}95 \text { por } 1000 \\
(60 \text { a } 147)\end{array}$ & $\begin{array}{c}\text { RR } 0,63 \\
(0,40 \text { a } 0,98)\end{array}$ & $\begin{array}{l}574 \\
(4)\end{array}$ & Baixa $^{2}$ & \\
\hline Satisfação do doente & \multicolumn{2}{|c|}{$\begin{array}{l}\text { Hospitalização domiciliária pode melhorar } \\
\text { ligeiramente a satisfação com cuidados de } \\
\text { saúde recebidos }\end{array}$} & - & $\begin{array}{l}795 \\
(6)\end{array}$ & Baixa $^{2}$ & \\
\hline $\begin{array}{l}\text { Duração do internamento } \\
\text { hospitalar }\end{array}$ & $\begin{array}{l}\text { Duração média de } \\
\qquad 16,1 \text { a } 42 \text { dias }\end{array}$ & $\begin{array}{c}\text { Duração média de } \\
-6,68 \text { dias } \\
(95 \% \text { IC }-10,19 \text { a -3,17) }\end{array}$ & $\begin{array}{c}\text { DM }-6,68 \\
(-10,19 a-3,17)\end{array}$ & $\begin{array}{l}528 \\
(4)\end{array}$ & Moderada $^{1}$ & $\begin{array}{l}\text { Outros } 5 \text { estudos } \\
\text { apresentaram uma } \\
\text { redução de } 8 \text { a } 15 \\
\text { dias }\end{array}$ \\
\hline Custo & \multicolumn{2}{|c|}{ Evidência incerta } & - & $\begin{array}{l}664 \\
(4)\end{array}$ & Muito baixa ${ }^{3}$ & \\
\hline
\end{tabular}

* O risco no grupo com intervenção (e o seu IC a 95\%) é baseado no risco assumido e no efeito relativo da intervenção (e do seu IC a 95\%).

AVC: acidente vascular cerebral; IC: intervalo de confiança; DM: diferença média; RR: risco relativo

Grupo de Trabalho GRADE (níveis de evidência):

- Qualidade alta: Temos muita confiança de que o verdadeiro efeito seja semelhante ao da estimativa do efeito.

- Qualidade moderada: Temos confiança moderada na estimativa do efeito: o verdadeiro efeito é provavelmente semelhante à estimativa do efeito, mas existe a possibilidade de ser substancialmente diferente.

- Qualidade baixa: A nossa confiança na estimativa do efeito é limitada: o verdadeiro efeito pode ser substancialmente diferente da estimativa do efeito.

- Qualidade muito baixa: Temos muito pouca confiança na estimativa do efeito: o verdadeiro efeito é provavelmente substancialmente diferente da estimativa do efeito.

1 - Redução de 1 ponto por imprecisão devido a IC alargado

2 - Redução de 2 pontos por imprecisão devido a IC alargado

3 - Redução de 3 pontos por inconsistência e imprecisão 
Tabela 2 - Sumário de resultados nos doentes com várias comorbilidades, adaptado da versão original

Efeito da alta precoce para hospitalização domiciliária em doentes com várias comorbilidades

Doente ou população: idosos com várias comorbilidades, que precisariam de cuidados em internamento hospitalar

Contexto: Austrália, Chile, Espanha, Holanda, Itália, Nova Zelândia, Turquia, Reino Unido

Intervenção: hospitalização domiciliária

Comparação: tratamento habitual

\begin{tabular}{|c|c|c|c|c|c|c|}
\hline \multirow[t]{2}{*}{ Resultados } & \multicolumn{2}{|c|}{$\begin{array}{l}\text { Riscos comparativos ilustrativos* } \\
\text { (IC } 95 \% \text { ) }\end{array}$} & \multirow[t]{2}{*}{$\begin{array}{l}\text { Efeito relativo } \\
\text { (IC 95\%) }\end{array}$} & \multirow{2}{*}{$\begin{array}{l}\text { Número de } \\
\text { participantes } \\
\text { (ensaios) }\end{array}$} & \multirow{2}{*}{$\begin{array}{l}\text { Qualidade da } \\
\text { evidência } \\
\text { (GRADE) }\end{array}$} & \multirow[t]{2}{*}{ Comentários } \\
\hline & $\begin{array}{l}\text { Ausência de alta } \\
\text { precoce para } \\
\text { hospitalização } \\
\text { domiciliária (risco } \\
\text { assumido) }\end{array}$ & $\begin{array}{l}\text { Alta precoce para } \\
\text { hospitalização } \\
\text { domiciliária } \\
\text { (risco } \\
\text { correspondente) }\end{array}$ & & & & \\
\hline
\end{tabular}

Doentes com várias comorbilidades (3 a 6 meses de seguimento)

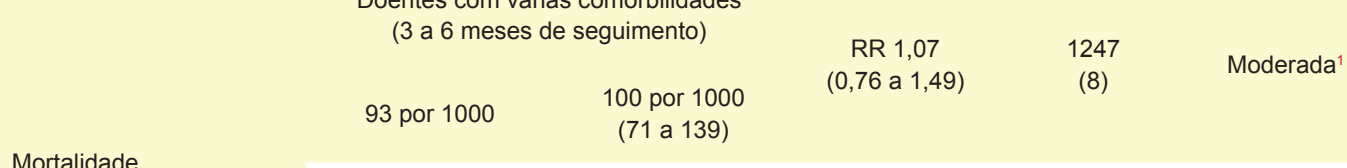

Mortalidade

Doentes com DPOC

(2 a 3 meses de seguimento)

35 por 1000
$\quad(17$ a 177$)$

RR 0,53

$(0,25$ a 1,12$)$

(17 a 177)

Doentes com várias comorbilidades

(3 meses de seguimento)

Reinternamento

hospitalar

$\begin{array}{rr}148 \text { por } 1000 \quad & (146 \text { a } 247)\end{array}$

Doentes com DPOC

(3 meses de seguimento)

$\begin{array}{cc}317 \text { por } 1000 \quad & 272 \text { por } 1000 \\ & (209 \text { a } 358)\end{array}$

Institucionalização ao fim de 1 ano

233 por 1000

161 por 1000
$(112$ a 231$)$

RR 0,69

$(0,48$ a 0,99$)$

RR 0,86

$(0,66$ a 1,13$)$

(5)

Baixa $^{2}$

RR 1,25

$(0,98$ a 1,58$)$

1276

(9)

Moderada

(2)

Alta precoce para hospitalização domiciliária pode melhorar ligeiramente a satisfação com os cuidados de saúde recebidos
Satisfação do doente
(5)
484

(3)
Baixa $^{2}$
Baixa $^{2}$

$\begin{array}{ll}900 & \text { Baixa }^{2}\end{array}$

\begin{tabular}{|c|c|c|c|c|}
\hline $\begin{array}{l}\text { Duração do internamento } \\
\text { hospitalar }\end{array}$ & $\begin{array}{l}\text { Nos doentes idosos a redução da duração do internamento } \\
\qquad \text { variou de }-20 \text { dias } a-12 \mathrm{~h}\end{array}$ & $\begin{array}{r}767 \\
(7)\end{array}$ & Moderada $^{1}$ & $\begin{array}{l}\text { Não foi realizado } \\
\text { analise combinada } \\
\text { por variação entre } \\
\text { estudos e ausência } \\
\text { de informação } \\
\text { relativamente ao } \\
\text { desvio padrão em } \\
\text { alguns estudos }\end{array}$ \\
\hline Custo & Evidência incerta & $\begin{array}{c}1369 \\
(8)\end{array}$ & Muito baixa ${ }^{3}$ & \\
\hline
\end{tabular}

* O risco no grupo com intervenção (e o seu IC a 95\%) é baseado no risco assumido e no efeito relativo da intervenção (e do seu IC a 95\%).

IC: intervalo de confiança; RR: risco relativo; DPOC: doença pulmonar obstrutiva crónica

Grupo de Trabalho GRADE (níveis de evidência):

- Qualidade alta: Temos muita confiança de que o verdadeiro efeito seja semelhante ao da estimativa do efeito.

- Qualidade moderada: Temos confiança moderada na estimativa do efeito: o verdadeiro efeito é provavelmente semelhante à estimativa do efeito, mas existe a possibilidade de ser substancialmente diferente.

- Qualidade baixa: A nossa confiança na estimativa do efeito é limitada: o verdadeiro efeito pode ser substancialmente diferente da estimativa do efeito.

- Qualidade muito baixa: Temos muito pouca confiança na estimativa do efeito: o verdadeiro efeito é provavelmente substancialmente diferente da estimativa do efeito.

1 - Redução de 1 ponto por imprecisão devido a IC alargado

2 - Redução de 2 pontos por imprecisão devido a IC alargado

3 - Redução de 3 pontos por inconsistência e imprecisão 
Tabela 3 - Sumário de resultados nos doentes após cirurgia, adaptado da versão original

\begin{tabular}{|c|c|c|c|c|c|c|}
\hline \multicolumn{7}{|c|}{ Efeito da alta precoce para hospitalização domiciliária em doentes em recuperação após cirurgia } \\
\hline \multicolumn{7}{|c|}{$\begin{array}{l}\text { Doente ou população: doentes em recuperação após cirurgia, que precisariam de cuidados em internamento hospitalar } \\
\text { Contexto: Austrália, Reino Unido, Suécia } \\
\text { Intervenção: hospitalização domiciliária } \\
\text { Comparação: tratamento habitual }\end{array}$} \\
\hline \multirow[t]{2}{*}{ Resultados } & \multicolumn{2}{|c|}{$\begin{array}{l}\text { Riscos comparativos ilustrativos* } \\
\text { (IC 95\%) }\end{array}$} & $\begin{array}{l}\text { Efeito relativo } \\
\text { (IC } 95 \%)\end{array}$ & $\begin{array}{l}\text { Número de } \\
\text { participantes } \\
\text { (ensaios) }\end{array}$ & $\begin{array}{l}\text { Qualidade da } \\
\text { evidência } \\
\text { (GRADE) }\end{array}$ & Comentários \\
\hline & $\begin{array}{l}\text { Ausência de alta } \\
\text { precoce para } \\
\text { hospitalização } \\
\text { domiciliária } \\
\text { (risco assumido) }\end{array}$ & $\begin{array}{l}\text { Alta precoce para } \\
\text { hospitalização } \\
\text { domiciliária (risco } \\
\text { correspondente) }\end{array}$ & & & & \\
\hline
\end{tabular}

\begin{tabular}{|c|c|c|c|c|c|}
\hline Mortalidade & \multicolumn{2}{|c|}{ Diferença reduzida ou ausente } & - & $\begin{array}{c}856 \\
(3)\end{array}$ & Baixa $^{1}$ \\
\hline Reinternamento hospitalar & \multicolumn{2}{|c|}{ Diferença reduzida ou ausente } & - & $\begin{array}{c}1229 \\
(5)\end{array}$ & Baixa $^{1}$ \\
\hline Institucionalização & \multicolumn{2}{|c|}{ Dados não reportados } & - & - & - \\
\hline Satisfação do doente & \multicolumn{2}{|c|}{$\begin{array}{l}\text { Alta precoce para hospitalização } \\
\text { domiciliária pode melhorar ligeiramente } \\
\text { a satisfação com os cuidados de saúde } \\
\text { recebidos }\end{array}$} & - & $\begin{array}{c}1229 \\
(5)\end{array}$ & Baixa $^{1}$ \\
\hline $\begin{array}{l}\text { Duração do internamento } \\
\text { hospitalar (doentes a } \\
\text { recuperar de cirurgia } \\
\text { ortopédica) }\end{array}$ & $\begin{array}{l}\text { Duração média de } \\
\text { internamento variou } \\
\text { de } 11,9 \text { a } 41,9 \text { dias }\end{array}$ & $\begin{array}{c}\text { Duração média de } \\
\text { internamento foi de } \\
-4,44 \text { dias } \\
(95 \% \text { IC }-6,37 \text { a }-2,51)\end{array}$ & $\begin{array}{c}\text { DM }-4,44 \\
(-6,37 \text { a }-2,51)\end{array}$ & $\begin{array}{l}411 \\
(4)\end{array}$ & Moderada $^{2}$ \\
\hline Custo & \multicolumn{2}{|c|}{ Evidência insuficiente } & - & $\begin{array}{c}1129 \\
(5)\end{array}$ & Muito baixa ${ }^{3}$ \\
\hline
\end{tabular}

* O risco no grupo com intervenção (e o seu IC a 95\%) é baseado no risco assumido e no efeito relativo da intervenção (e do seu IC a 95\%).

IC: intervalo de confiança; DM: diferença média; RR: risco relativo

Grupo de Trabalho GRADE (níveis de evidência):

- Qualidade alta: Temos muita confiança de que o verdadeiro efeito seja semelhante ao da estimativa do efeito.

- Qualidade moderada: Temos confiança moderada na estimativa do efeito: o verdadeiro efeito é provavelmente semelhante à estimativa do efeito, mas existe a possibilidade de ser substancialmente diferente.

- Qualidade baixa: A nossa confiança na estimativa do efeito é limitada: o verdadeiro efeito pode ser substancialmente diferente da estimativa do efeito.

- Qualidade muito baixa: Temos muito pouca confiança na estimativa do efeito: o verdadeiro efeito é provavelmente substancialmente diferente da estimativa do efeito.

1 - Redução de 2 ponto por imprecisão devido a IC alargado

2 - Redução de 1 pontos por imprecisão devido a IC alargado

3 - Redução de 3 pontos por inconsistência e imprecisão

fisioterapeutas, terapeutas ocupacionais e assistentes sociais). Num estudo de reabilitação o apoio era prestado por voluntários da Cruz Vermelha. Os principais resultados encontram-se nas Tabelas 1 a 3.

\section{CONCLUSÃO}

Apesar do crescente interesse no potencial de serviços de hospitalização domiciliária como uma alternativa menos dispendiosa ao internamento hospitalar, a presente revisão identificou que a evidência existente sobre o benefício clínico e económico (através de uma redução no período de internamento hospitalar) desta intervenção é insuficiente.

\section{COMENTÁRIO}

Hospital at home ou hospitalização domiciliária constitui um modelo inovador que providencia cuidados de saúde hospitalares no domicílio, em substituição dos cuidados prestados habitualmente em internamento hospitalar. ${ }^{1,2}$ Foi testado por vários centros médicos em diversos países, encontrando-se a ser desenvolvido também em alguns centros portugueses (ex: Hospital Garcia da Orta)., ${ }^{2,3}$

Este modelo resultou da necessidade de gerir um recurso limitado que são as camas de internamento de curta duração no hospital. No entanto, é importante avaliar se esta mudança no tratamento não acarreta aumento de risco dos cuidados clínicos prestados durante o episódio agudo, ou posteriormente no número de readmissões hospitalares e institucionalizações. Outro fator importante a ser analisado é a potencial vantagem económica na redução dos custos do internamento hospitalar. ${ }^{1}$

Os autores da revisão sistemática pretenderam avaliar o efeito da hospitalização domiciliária na mortalidade e reinternamento hospitalar nos 3 a 6 meses após o evento agudo. Paralelamente, foi avaliada a institucionalização, bem como a satisfação dos doentes, duração de internamento e custos em saúde. ${ }^{1}$

Os autores concluíram que a evidência existente é incerta relativamente ao benefício económico ou melhoria 
dos resultados clínicos na utilização de recursos de saúde com a hospitalização domiciliária. No entanto, esta revisão conclui, com grau de evidência baixo a moderado, que estes cuidados de saúde não afetam a mortalidade, reinternamento hospitalar ou status funcional e pode melhorar ligeiramente a satisfação dos doentes. ${ }^{1}$ Deste modo, a hospitalização domiciliária parece poder ser uma opção válida, mas necessitamos de mais informação sobre o impacto desta medida.

Atendendo ao envelhecimento da população e ao crescente consumo de cuidados de saúde pela população idosa, é desejável a continuação da realização de ensaios clínicos nesta população de doentes de modo a avaliar o impacto de diferentes intervenções (ex: hospitalização domiciliária) nos custos em saúde.

\section{IMPLICAÇÕES PARA A PRÁTICA CLÍNICA}

- A hospitalização domiciliária é um método válido para reduzir a pressão de utilização de camas de internamento hospitalar.

- A hospitalização domiciliária demonstrou, com evidência de baixa a moderada qualidade, não alterar a mortalidade, reinternamento hospitalar ou status funcional.

\section{REFERÊNCIAS}

1. Gonçalves-Bradley DC, lliffe S, Doll HA, Broad J, Gladman J, Langhorne $P$, et al. Early discharge hospital at home. Cochrane Database Syst Rev. 2017;6:CD000356.

2. Alves M. "Hospital at Home": The reality inside and outside of Portugal.

Rev Soc Port Med Interna. 2016;23:40-3

3. Administração Regional de Saúde de Lisboa e Vale do Tejo [consultado 2017 ago 11]. Disponivel em: http://www.arslvt.min-saude.pt/frontoffice/ pages/2?news_id=853.

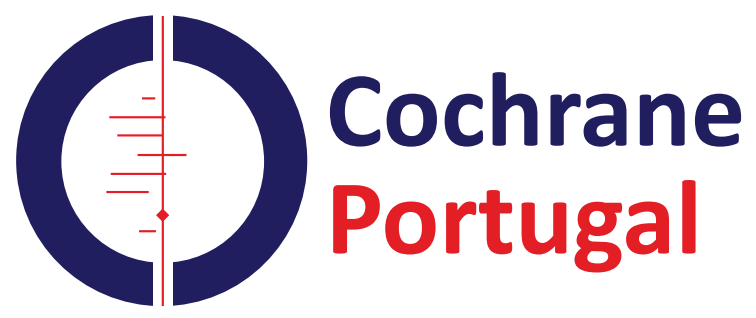

